\title{
Parity-Time Synthetic Phononic Media
}

\author{
Christensen, Johan; Willatzen, Morten; Velasco, V. R.; Lu, M.-H.
}

\section{Published in:}

Physical Review Letters

Link to article, DOI:

10.1103/PhysRevLett.116.207601

Publication date:

2016

\section{Document Version}

Publisher's PDF, also known as Version of record

Link back to DTU Orbit

Citation (APA):

Christensen, J., Willatzen, M., Velasco, V. R., \& Lu, M-H. (2016). Parity-Time Synthetic Phononic Media. Physical Review Letters, 116, [207601]. https://doi.org/10.1103/PhysRevLett.116.207601

\section{General rights}

Copyright and moral rights for the publications made accessible in the public portal are retained by the authors and/or other copyright owners and it is a condition of accessing publications that users recognise and abide by the legal requirements associated with these rights.

- Users may download and print one copy of any publication from the public portal for the purpose of private study or research.

- You may not further distribute the material or use it for any profit-making activity or commercial gain

- You may freely distribute the URL identifying the publication in the public portal

If you believe that this document breaches copyright please contact us providing details, and we will remove access to the work immediately and investigate your claim 


\title{
Parity-Time Synthetic Phononic Media
}

\author{
J. Christensen, ${ }^{1, *}$ M. Willatzen, ${ }^{1}$ V. R. Velasco, ${ }^{2}$ and M.-H. $\mathrm{Lu}^{3}$ \\ ${ }^{1}$ Department of Photonics Engineering, Technical University of Denmark, DK-2800 Kongens Lyngby, Denmark \\ ${ }^{2}$ Instituto de Ciencia de Materiales de Madrid, (ICMM,CSIC), Sor Juana Inés de la Cruz 3, 28049 Madrid, Spain \\ ${ }^{3}$ National Laboratory of Solid State Microstructures and Department of Materials Science and Engineering, \\ Nanjing University, Nanjing 210093, China
}

(Received 9 January 2016; published 19 May 2016)

\begin{abstract}
Classical systems containing cleverly devised combinations of loss and gain elements constitute extremely rich building units that can mimic non-Hermitian properties, which conventionally are attainable in quantum mechanics only. Parity-time $(\mathcal{P} \mathcal{T})$ symmetric media, also referred to as synthetic media, have been devised in many optical systems with the ground breaking potential to create nonreciprocal structures and one-way cloaks of invisibility. Here we demonstrate a feasible approach for the case of sound where the most important ingredients within synthetic materials, loss and gain, are achieved through electrically biased piezoelectric semiconductors. We study first how wave attenuation and amplification can be tuned, and when combined, can give rise to a phononic $\mathcal{P} \mathcal{T}$ synthetic media with unidirectional suppressed reflectance, a feature directly applicable to evading sonar detection.
\end{abstract}

DOI: 10.1103/PhysRevLett.116.207601

$\mathcal{P} \mathcal{T}$ symmetric systems have recently attracted tremendous attention in the study of extraordinary physics with non-Hermitian Hamiltonians. A distinctive aspect of $\mathcal{P} \mathcal{T}$ symmetry is that such a system remains unaffected after space-time reflection. Through cunningly synthesized media containing balanced loss and gain units, these structures disobey parity symmetry and time-reversal symmetry, but are nevertheless symmetric under simultaneous operation [1-5]. In order to realize $\mathcal{P} \mathcal{T}$ symmetric Hamiltonians there are distinctive blueprints to follow. In nonrelativistic quantum mechanics, governed by the Schrödinger equation, complex potentials must be involved, possessing real and imaginary parts that are even and odd functions of position, respectively. The same is true in optics following this prescription with a complex index of refraction [6-8].

The novel approach based on $\mathcal{P} \mathcal{T}$ symmetry is to manipulate absorption using judicious structure designs with gain regions that exhibit intriguing properties as we will see in the following. The Hamiltonian of such structures commutes with the combined $\mathcal{P} \mathcal{T}$ operator and gives rise to entirely real energy solutions. As long as these eigensolutions are real, it is said that the system is within an exact $\mathcal{P} \mathcal{T}$ symmetric phase. In this phase, power oscillation occurs, but most remarkably, nonreciprocal wave propagation sets in $[3,5,9]$. When loss or gain increases the eigenvalues become complex as happens within a broken $\mathcal{P} \mathcal{T}$ symmetric phase. In this scenario, the wave dynamics is nonreciprocal with eigenvalues that appear in complex-conjugate pairs, resulting in some of the modes experiencing either an exponential increase (amplifier) or decrease (absorber) in total power. The resulting behavior is both the one of a laser oscillator emitting in-phase waves and of a coherent perfect absorber that fully absorbs incoming light [6,10-12]. Exactly at the $\mathcal{P} \mathcal{T}$ phase transition point, the two eigenvalues coalesce leading to an exceptional point singularity. At that abrupt phase transition the transmission becomes unitary but the system, in view of the reflection, attains perfect nonreciprocity. In other words, such a system is highly reflective when probed from one end but perfectly transparent (zero reflection) when irradiated at the opposite end [4,5].

These fascinating properties with common notions from quantum mechanics have important applications for classical fields such as optics and acoustics in view of one-way mirrors, cloaks of invisibility and coherent laser absorbers. In order to realize such non-Hermitian $\mathcal{P} \mathcal{T}$ symmetric systems for sound waves, phononic structures have to be synthesized that contain active lossy constituents in combination with the time-reversed image, that is, the gain counterpart. First examples have already been realized based on optomechanical and electromechanical devices [13-15]. More specifically, although Zhu et al. studied intriguing effects associated with $\mathcal{P} \mathcal{T}$ symmetry, no existing gain media were used [13]. Fleury et al., on the other hand, used actively loaded loudspeaker systems [14]. Acoustic diodes have been achieved by combining a nonlinear material with gain and lossy materials [16]. In this Letter, we propose a continuum mechanical approach to realize phononic $\mathcal{P} \mathcal{T}$ symmetry through the intrinsic acoustoelectric effect in piezoelectric semiconductors. Instead of using rather complex transducer devices and electronic circuits, piezoelectric semiconductors constitute, by themselves, solid state materials capable of amplifying sound waves. Using these materials, we show that one is able to actively control the elastic wave response through electric bias, resulting in either sound wave attenuation or amplification, which are the basic ingredients needed to 
synthesize phononic $\mathcal{P} \mathcal{T}$ symmetry. As we mentioned earlier, optics offers a base to experimentally realize and test properties associated with non-Hermitian $\mathcal{P} \mathcal{T}$ systems. The reason is the formal equivalence between the Schrödinger equation and the optical wave equation. How does this translate to sound waves? The stationary Schrödinger equation reads

$$
\frac{\partial^{2} \psi(z)}{\partial z^{2}}-\frac{2 m}{\hbar^{2}}[V(z)-E] \psi(z)=0,
$$

where $\psi(z)$ is the wave function, $E$ is the energy, and $V(z)$ is the complex potential. The condition for $\mathcal{P} \mathcal{T}$ symmetry in a quantum mechanical system requires the complex potential to satisfy the condition $V(z)=V^{*}(-z)$. If we undertake a few substitutions in Eq. (1), $V(z)-E \rightarrow$ $\left[\omega^{2} \rho(z) / c_{33}^{\prime}(z)\right], \psi(z) \rightarrow u(z)$, and $-\left(2 m / \hbar^{2}\right) \rightarrow 1$, we arrive at an equation of the following form for longitudinal vibrations in wurtzite structures with hexagonal $6 \mathrm{~mm}$ symmetry (refer to the Supplemental Material [17]):

$$
\frac{\partial^{2} u}{\partial z^{2}}+\frac{\omega^{2} \rho(z)}{c_{33}^{\prime}(z)} u=0
$$

Equation (2) is the 1D elastic wave equation for longitudinal fields where $u(z)$ is the displacement along $z, \rho$ is the mass density, and $c_{33}^{\prime}$ is the effective stiffness tensor 33 component [refer to Eq. (4) for further details]. In this context, we can write the conditions for the synthesis of $\mathcal{P} \mathcal{T}$ phononic media using realistic materials

$$
\rho(z)=\rho(-z), c_{33}^{\prime}(z)=c_{33}^{*}(-z) .
$$

These requirements suggest that for real, i.e., lossy materials, an elastic gain compensation is inevitably needed as described by the imaginary part of the effective stiffness. A possibility to amplify sound waves is facilitated through the acoustoelectric effect. When an acoustic field impinges on a piezoelectric semiconductor slab a coherently oscillating electric charge is created. Superimposing a sufficiently high dc electric field $E_{0}$ corresponding to a supersonic carrier drift speed $\left(v_{d}>v_{s}\right)$ leads to sound amplification by virtue of phonon emission, an effect known as acoustic Cherenkov radiation [18,19]. The velocity $v_{s}=\omega / k$ is the sound speed of the semiconductor with wave number $k$ and $v_{d}=\mu_{33} E_{0}$ corresponds to the drift velocity with the mobility $\mu_{33}$ [20]. Amplification of sound was first observed by Hutson et al. in $\mathrm{CdS}$ and recently also investigated in connection with surface wave amplification and directional control in graphene and $\mathrm{GaN}$ [21-23]. The effective stiffness of the piezoelectric semiconductor is written in the following form: [18]

$$
\frac{c_{33}^{\prime}}{c_{33}}=1+K^{2} \frac{\gamma+i \frac{\omega}{\omega_{d}}}{\gamma+i\left(\frac{\omega_{c}}{\omega}+\frac{\omega}{\omega_{d}}\right)},
$$

where $\gamma=1-v_{d} / v_{s}$ is a drift parameter, whereas $\omega_{c}=$ $\sigma / \varepsilon_{33}$ and $\omega_{d}=v_{s} / d_{33}$ represent the dielectric relaxation and the diffusion frequency, respectively, where $\varepsilon_{33}$ is the permittivity, $\sigma$ the conductivity, and $d_{33}$ the carrier diffusion constant.

We begin the study by considering piezoelectric wurtzite structures whose mechanical response, when waves are longitudinally polarized, has to fulfill the condition given in Eq. (3). Various materials can be employed such as GaAs, $\mathrm{GaN}$, and InSb but we restrict our study to $\mathrm{ZnO}$ for its low conductivity that keeps the drift currents low and for the relatively small intrinsic plasma frequency that is important for mechanical applications. It is possible, at moderate amplification and damping levels, to express the attenuation coefficient as [19]

$$
\alpha=\frac{K^{2}}{2} \frac{\omega}{v_{s}} \frac{\gamma \frac{\omega_{c}}{\omega}}{\gamma^{2}+\left(\frac{\omega_{c}}{\omega}+\frac{\omega}{\omega_{d}}\right)^{2}} .
$$

In Eq. (5) $K=e_{33} / \sqrt{c_{33} \varepsilon_{33}}$ quantifies the piezoelectric coupling, where $e_{33}$ is the piezoelectric constant and $c_{33}$ the stiffness of the hexagonal crystal. As one can see in Fig. 1, the negative (positive) part of the attenuation coefficient $\alpha$ corresponds to phonon amplification (damping) whose magnitude around the Cherenkov threshold can be controlled through the electric bias $E_{0}$. We mentioned earlier that systems respecting the space-time reflection symmetry rely on the combined effects of gain and loss. Figures 1(a) and 1(b) show how these essential features can be tuned and spectrally shifted by modifying the carrier concentration $n$ (normalized to the intrinsic concentration $n_{i}$ ) and changing the frequency $\omega$ (normalized to the plasma frequency $\omega_{p}=\sqrt{n q^{2} / \varepsilon_{\infty} \varepsilon_{0} m_{\mathrm{eff}}}$, where $q$ is the electric charge, $m_{\text {eff }}$ the effective mass, whereas $\varepsilon_{\infty}$ and $\varepsilon_{0}$ are the high frequency and vacuum permittivity), respectively. When a dc field is applied, the elastic displacement will follow a stationary motion and one that varies in time. We only consider the dynamic one as it suffices for the boundary value problem that will follow. The elastic wave motion $u(\omega)$ parallel to the applied electric field and the normal stress $T_{z z}(\omega)$ are written as time-dependent fields

$$
\begin{aligned}
u(\omega) & =\sum_{n=1}^{2} \delta_{n} \phi_{n} e^{i k_{n} z} e^{i \omega t}, \\
T_{z z}(\omega) & =\sum_{n=1}^{2} \eta_{n} \phi_{n} e^{i k_{n} z} e^{i \omega t} .
\end{aligned}
$$

In Eq. (6) the electric potential $\phi_{n}$ is related to the displacement via $\delta_{n}$ and to the stress via $\eta_{n}$

$$
\begin{aligned}
\delta_{n} & =-\frac{e_{33} k_{n}^{2}}{c_{33} k_{n}^{2}-\rho \omega^{2}}, \\
\eta_{n} & =\frac{k_{n}}{\omega \sqrt{\rho^{B} c_{33}^{B}}}\left(\delta_{n} c_{33}+e_{33}\right),
\end{aligned}
$$




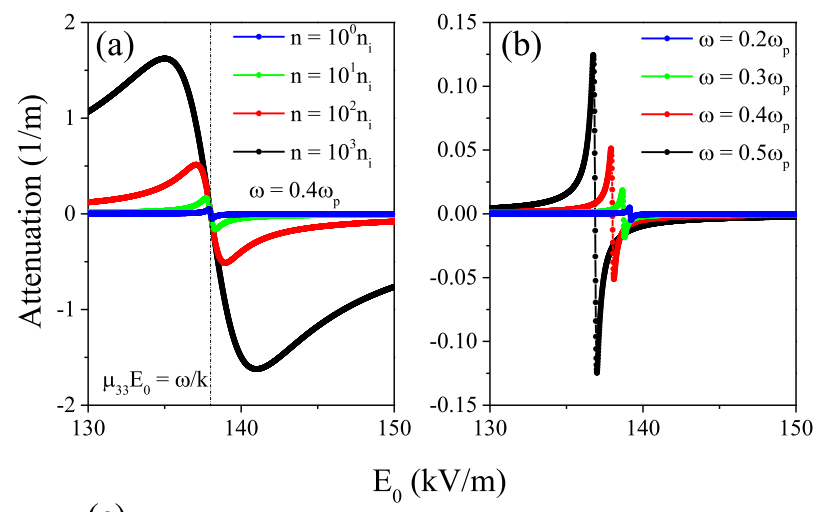

(c)

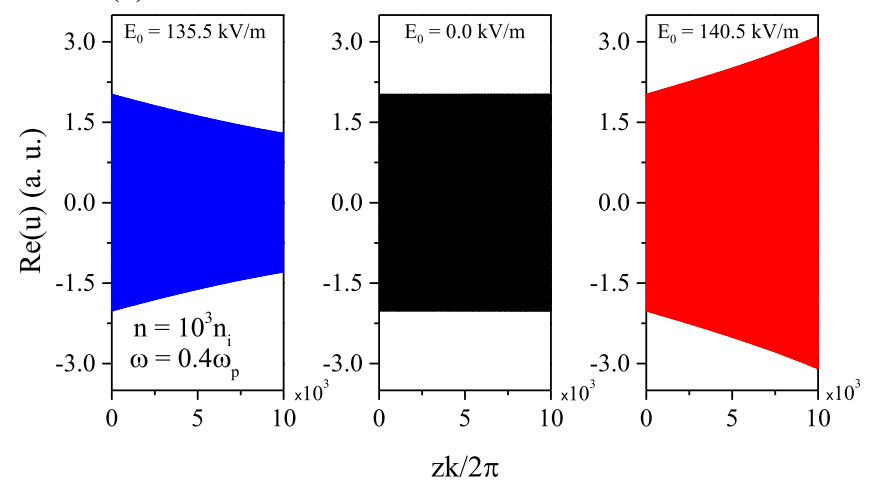

FIG. 1. Gain or attenuation of sound in $\mathrm{ZnO}$ at (a) various carrier concentrations $n / n_{i}$ and for (b) different frequencies. The vertical dashed-dotted line marks the Cherenkov threshold $\left(v_{d}=v_{s}\right)$. In panel (b) $n=n_{i}$ with a plasma frequency at $9 \mathrm{MHz}$. (c) From (a) we take the example where $n=10^{3} n_{i}$ corresponding to a plasma frequency at $280 \mathrm{MHz}$ and plot the elastic displacements $u$ at the gain or attenuation peaks and where the electric bias is switched off, $E_{0}=0$.

normalized to reference parameters $\rho^{B}$ and $c_{33}^{B}$. We employ an exact model that describes the piezoelectric interaction in $\mathrm{ZnO}$ to second-order accuracy in the mobility $\mu_{33}$ [20]. This theory is well suited in a small signal approximation. In the bulk of $\mathrm{ZnO}$ we obtain two modal solutions with wave numbers denoted $k_{1}$ and $k_{2}$ (refer to the Supplemental Material [17]). These solutions correspond to one piezoelectric mode propagating along the electric field direction and one opposite to it. Note that when the piezoelectric media is not activated $E_{0}=0$ and $k_{1}=-k_{2}=\omega / v_{s}$. In the unbound $\mathrm{ZnO}$ bulk, we take $\phi_{n}=1$ and compute $u(\omega)$ from Eq. (6) with applied electric fields as indicated in Fig. 1(c), corresponding to phononic attenuation, free propagation, and gain. These calculations show that we indeed are able to synthesize PT symmetry for sound based on the intrinsic piezoelectricity in semiconductor materials.

In order to satisfy the strict requirements of $\mathcal{P} \mathcal{T}$ symmetry the conditions given in Eq. (3) must be fulfilled. In Fig. 2 we plot Eq. (4) as a function of $E_{0}$ and the drift-to-sound velocity ratio. At the Cherenkov threshold $\operatorname{Im}\left\{c_{33}^{\prime}\right\}=0$. Detuning the phononic response below or above this point

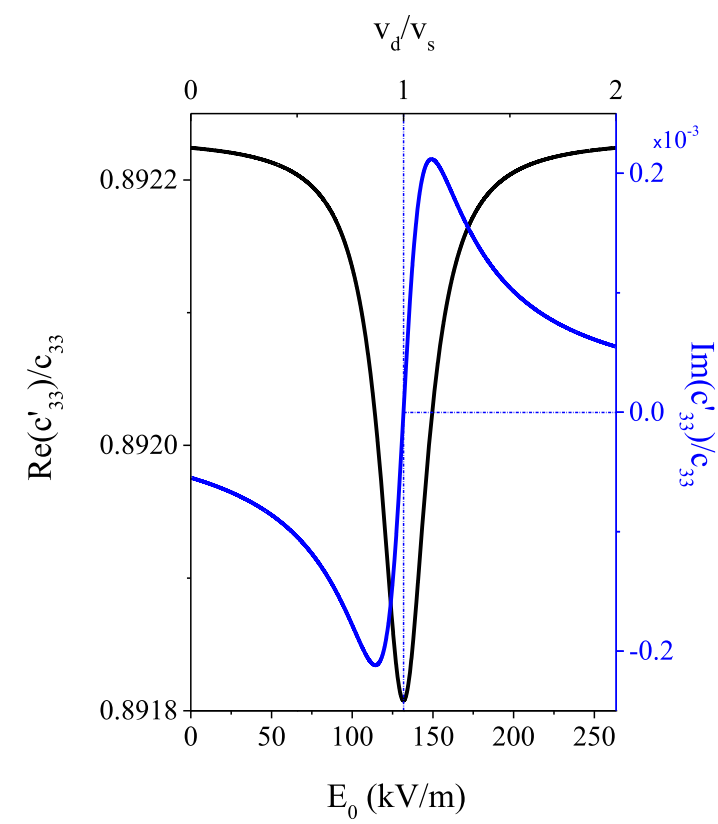

FIG. 2. $\quad c_{33}^{\prime}$ is computed versus $E_{0}$ for $\mathrm{ZnO}$ with $n=10^{4} n_{i}$ at $\omega=0.7 \omega_{p}$. Top scale represents the data as a function of $v_{d} / v_{s}$. Left(right) axis represents the real(imaginary) part of $c_{33}^{\prime}$. At the Cherenkov threshold, $v_{d}=v_{s}$ (dash-dotted line at $E_{0}=131.8 \mathrm{kV} / \mathrm{m}$ ), the effective stiffness $c_{33}^{\prime}$ is purely real.

gives rise to either a negative or positive effective imaginary stiffness $c_{33}^{\prime}$ which is similar, but of opposite sign, to the behavior of the attenuation coefficient in Fig. 1. Note that $\operatorname{Re}\left\{c_{33}^{\prime}\right\}$ and $\left|\operatorname{Im}\left\{c_{33}^{\prime}\right\}\right|$ are symmetric and therefore balanced around the Cherenkov threshold.

As we will see in the following, even when the $\mathcal{P} \mathcal{T}$ condition can be met (Fig. 2), it must be borne in mind that gain and loss are deterministically controlled only when sound propagates and electrons drift along the same path. $\mathcal{P} \mathcal{T}$ symmetry based on the electroacoustic effect is therefore challenging because gain or loss does not obey timereversal symmetry since the scattering matrix $S$ for a biased $\mathrm{ZnO}$ slab always will be asymmetric, $S_{n m} \neq S_{m n}$. To show that, we compute the scattering parameters for various biased $\mathrm{ZnO}$ slabs at different drift levels according to Fig. 2. For a single layer we derive analytical expressions for these coefficients

$$
\begin{aligned}
& S_{11}=\frac{2}{D}\left[-\delta_{2}\left(\delta_{1}+\eta_{1}\right) e^{i k_{1} h}+\delta_{1}\left(\delta_{2}+\eta_{2}\right) e^{i k_{2} h}\right]-1, \\
& S_{21}=\frac{2}{D}\left[-\delta_{2}\left(\delta_{1}+\eta_{1}\right)+\delta_{1}\left(\delta_{2}+\eta_{2}\right)\right] e^{i\left(k_{1}+k_{2}\right) h}, \\
& S_{22}=\frac{2}{D}\left[\delta_{2}\left(\delta_{1}-\eta_{1}\right) e^{i k_{2} h}-\delta_{1}\left(\delta_{2}-\eta_{2}\right) e^{i k_{1} h}\right]-1, \\
& S_{12}=\frac{2}{D}\left[\delta_{2}\left(\delta_{1}-\eta_{1}\right)-\delta_{1}\left(\delta_{2}-\eta_{2}\right)\right],
\end{aligned}
$$

where $D=\left(\delta_{1}-\eta_{1}\right)\left(\delta_{2}+\eta_{2}\right) e^{i k_{2} h}-\left(\delta_{1}+\eta_{1}\right)\left(\delta_{2}-\eta_{2}\right) e^{i k_{1} h}$. In the first scenario, a voltage is applied with an electric 
field of $E_{G}=150.0 \mathrm{kV} / \mathrm{m}$ across the slab. This field generates phonon amplification with $\alpha=-71 \mathrm{~m}^{-1}$ (at $\left.\operatorname{Im}\left\{c_{33}^{\prime}\right\}=0.21 \times 10^{-3} c_{33}\right)$ in forward direction and $\alpha=$ $8.75 \mathrm{~m}^{-1}\left(\right.$ at $\left.\operatorname{Im}\left\{c_{33}^{\prime}\right\}=-0.26 \times 10^{-4} c_{33}\right)$ in the backward direction. We predict equivalent $\left|S_{11}\right|^{2}=\left|S_{22}\right|^{2}$ reflectance at both slab sides in all examples but the single gain slab exhibits a strong asymmetry between both transmission channels with increasing frequency. Sound propagation against the drift flow corresponds to $v_{d} / v_{s}<0$ and produces wave attenuation only (not plotted here). When the electrons drift symmetrically towards the slab center coming in from both sides time-reversal symmetry is preserved as the scattering parameters spectra show in the middle panel. This setup is a neat way to circumvent the inherent nonreciprocity. This necessity is illustrated by the last example where we have balanced loss and gain in forward direction only, $E_{L}=113.5 \mathrm{kV} / \mathrm{m}$ with

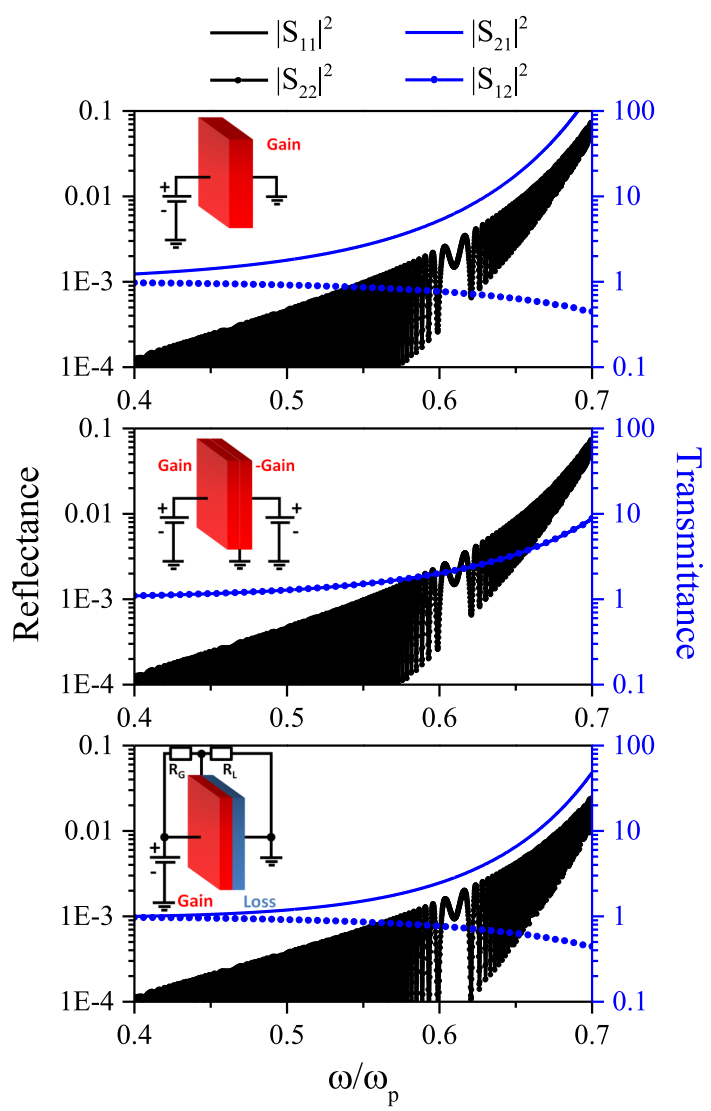

FIG. 3. Scattering parameters for various $\mathrm{ZnO}$ slab setups biased with a voltage source. In all cases, the overall slab thickness $h=4 \mathrm{~cm}$. Upper panel: Slab is biased in forward direction compared to the sound direction (left to right), $E_{G}=150.0 \mathrm{kV} / \mathrm{m}$. Middle panel: Bias in a bidirectional fashion with $E_{G}=150.0 \mathrm{kV} / \mathrm{m}$ and $E_{-G}=-E_{G}$. Lower panel: Balanced loss and gain through a voltage divider in forward direction, $E_{G}=150.0 \mathrm{kV} / \mathrm{m}$ and $E_{L}=113.5 \mathrm{kV} / \mathrm{m}$. In the simulations the background is impedance matched to $\mathrm{ZnO}$, hence, $\rho^{B}=\rho$ and $c_{33}^{B}=c_{33}$. $\alpha=71 \mathrm{~m}^{-1}$ but $\alpha=10.04 \mathrm{~m}^{-1}$ in the opposite direction. Although we intentionally balanced the amount of loss and gain with the necessary electric bias, the system in the last example of Fig. 3 does not obey $\mathcal{P} \mathcal{T}$ symmetry since the overall energy flow is not controlled and balanced for forward and backward sound propagation. This challenge makes it somewhat difficult to smoothly tune the amount of loss and gain from within an exact towards a broken $\mathcal{P} \mathcal{T}$ phase.

Loading the gain and loss components symmetrically as illustrated in Fig. 4(a) produces time-reversal symmetric amplification and attenuation in each constituent. However, as loss dominates since it is inevitably present in all backward directed flow $\left(v_{d} / v_{s}<0\right)$, we will tune towards $\mathcal{P} \mathcal{T}$ symmetry with zero net attenuation by steadily increasing the phononic gain as plotted in Fig. 4(b). To obtain the overall scattering parameters for the binary system shown in Fig. 4(a), we implemented calculations involving a rigorous expansion, both in terms of radiating
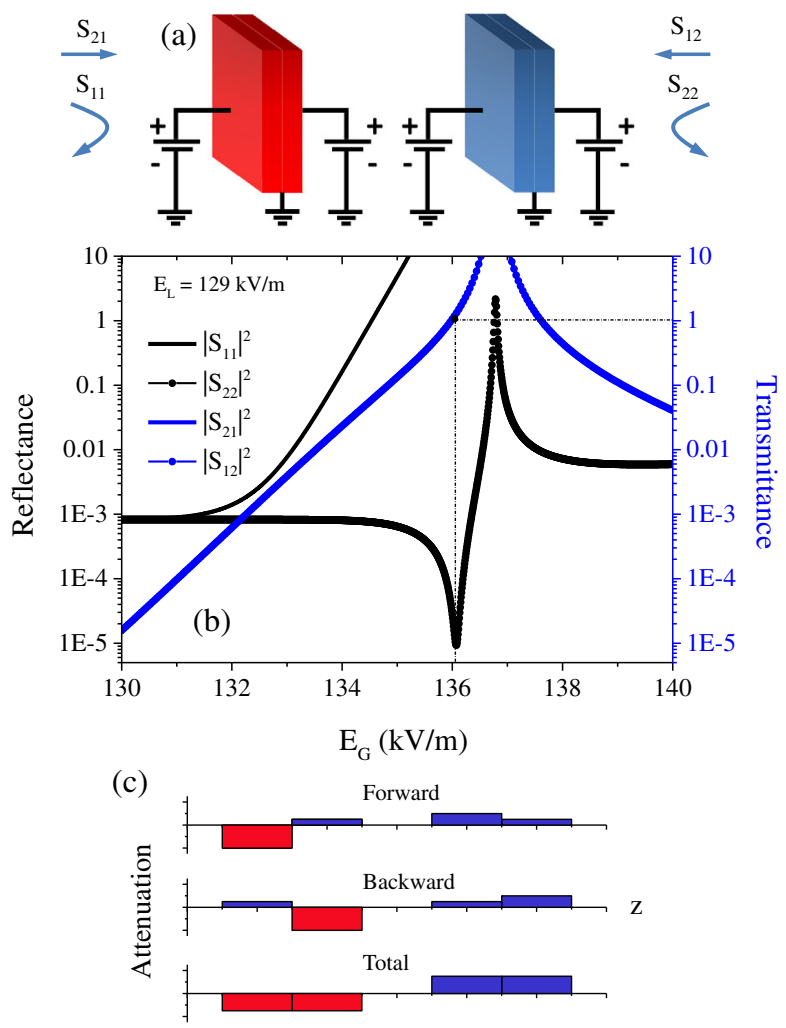

FIG. 4. (a) $\mathcal{P} \mathcal{T}$ symmetric binary system where the gain (red) and loss (blue) constituents are biased in an bidirectional fashion. $h=4 \mathrm{~cm}$ and the passive nonpiezoelectric separator region, with $\rho^{B}=\rho$ and $c_{33}^{B}=c_{33}$, has thickness $s=0.5 \mathrm{~mm}$. (b) Scattering parameters spectrum is plotted around the exceptional point (dashed line) as a function of $E_{g}$, whereas $E_{L}$ is locked. Same parameters are taken as in Fig. 2. (c) $\alpha$ is given for the $\mathcal{P} \mathcal{T}$ symmetry structure in (a) along the stacking direction $z$ when sound propagates forward, backwards, and after a round trip. 
waves on either side of the structure and waveguide modes within the biased slabs [17]. We lock the amount of loss in the right blue $\mathrm{ZnO}$ slabs at $E_{L}=129.0 \mathrm{kV} / \mathrm{m}$ and $E_{-L}=$ $-E_{L}$ such that loss after an acoustic round-trip is fully balanced, see Fig. 4(c). Then we raise the amount of gain in the left half of the binary structure by slowly increasing $E_{-G}=-E_{G}$ up to the point where the imaginary eigenvalues merge toward zero, or as illustrated in Fig. 4(b), a bidirectional transparent mode sets in which experiences zero reflection when probed from one end, $\left|S_{22}\right|^{2} \rightarrow 0$, but strong reflection when probed in the reverse direction, $\left|S_{11}\right|^{2}>1$. This location where the transmittance becomes unitary, $\left|S_{21}\right|^{2}=\left|S_{12}\right|^{2}=1$, corresponding to perfect nonreciprocity in the reflectance is referred to as the exceptional point [marked with the dotted line in Fig. 4(b)]. It is rather difficult to demonstrate the properties of $\mathcal{P} \mathcal{T}$ symmetry in its various phases around the exceptional point since gain and loss cannot be increased smoothly and simultaneously in a uniform manner. Figure 4(c) illustrates how the attenuation subsequently balances out towards zero net gain and loss when tuned at the exceptional point, $E_{G} \approx 136.0 \mathrm{kV} / \mathrm{m}$. Unidirectional suppression of the reflectance is the cornerstone of $\mathcal{P} \mathcal{T}$ symmetry media and the basic feature in order to design a one-way acoustic cloak of invisibility [24]. This unique property has been demonstrated in Fig. 4(b).

In summary, we have demonstrated $\mathcal{P} \mathcal{T}$ symmetry in piezoelectric semiconductors and explored the tunability through simple electric biasing. Since gain and loss in these media are strictly directional dependent the acoustic realization is a nontrivial extension from its optical counterpart. Our simulations predict that phononic $\mathcal{P} \mathcal{T}$ symmetry creates strong nonreciprocal reflections from either end of the structure and that these findings could advance the progress on, e.g., acoustic camouflage coatings for hiding submarines from sonar detection. Additional features associated to the $\mathcal{P} \mathcal{T}$ symmetry phases such as the coexistence of absorbing and lasing states for sound are yet to be explored. The realization of $\mathcal{P} \mathcal{T}$ non-Hermitian phononic lattices with peculiar scattering characteristics is another avenue worth investigating.

J. C. gratefully acknowledges financial support from the Danish Council for Independent Research and a Sapere Aude Grant (No. 12-134776). V. R. V. gratefully acknowledges financial support from the Spanish Ministerio de Economía y Competitividad through Grant No. MAT201566888-C3-1-R.
* Corresponding author. johan.christensen@gmail.com

[1] C. M. Bender and S. Boettcher, Phys. Rev. Lett. 80, 5243 (1998).

[2] H. Schomerus, Phys. Rev. Lett. 104, 233601 (2010).

[3] C. E. Ruter, K. G. Makris, R. El-Ganainy, D. N. Christodoulides, M. Segev, and D. Kip, Nat. Phys. 6, 192 (2010).

[4] Z. Lin, H. Ramezani, T. Eichelkraut, T. Kottos, H. Cao, and D. N. Christodoulides, Phys. Rev. Lett. 106, 213901 (2011).

[5] A. Regensburger, C. Bersch, M. A. Miri, G. Onishchukov, D. N. Christodoulides, and U. Peschel, Nature (London) 488, 167 (2012).

[6] Y. D. Chong, L. Ge, H. Cao, and A. D. Stone, Phys. Rev. Lett. 105, 053901 (2010).

[7] L. Ge, Y. D. Chong, and A. D. Stone, Phys. Rev. A 85, 023802 (2012).

[8] Y. Sun, W. Tan, H. Q. Li, J. Li, and H. Chen, Phys. Rev. Lett. 112, 143903 (2014).

[9] B. Peng, Ş. K. Özdemir, F. Lei, F. Monifi, M. Gianfreda, G. L. Long, S. Fan, F. Nori, C. M. Bender, and L. Yang, Nat. Phys. 10, 394 (2014).

[10] S. Longhi, Phys. Rev. A 82, 031801(R) (2010).

[11] L. Feng, Z. J. Wong, R.-M. Ma, Y. Wang, and X. Zhang, Science 346, 972 (2014).

[12] H. Hodaei, M.-A. Miri, M. Heinrich, D. N. Christodoulides, and M. Khajavikhan, Science 346, 975 (2014).

[13] X. Zhu, H. Ramezani, C. Shi, J. Zhu, and X. Zhang, Phys. Rev. X 4, 031042 (2014).

[14] R. Fleury, D. L. Sounas, and A. Alu, Nat. Commun. 6, 5905 (2015).

[15] X.-W. Xu, Y.-X. Liu, C.-P. Sun, and Y. Li, Phys. Rev. A 92, 013852 (2015).

[16] Z.-m. Gu, J. Hu, B. Liang, X.-y. Zou, and J.-c. Cheng, Sci. Rep. 6, 19824 (2016).

[17] See Supplemental Material at http://link.aps.org/ supplemental/10.1103/PhysRevLett.116.207601 for a discussion on the time-reversal symmetry breaking and details on the numerical implementation of the PT symmetric binary system.

[18] A. R. Hutson, J. H. McFee, and D. L. White, Phys. Rev. Lett. 7, 237 (1961).

[19] D. L. White, J. Appl. Phys. 33, 2547 (1962).

[20] J. Christensen and M. Willatzen, Acust. Acta Acust. 101, 986 (2015).

[21] Z. Insepov, E. Emelin, O. Kononenko, D. V. Roshchupkin, K. B. Tnyshtykbayev, and K. A. Baigarin, Appl. Phys. Lett. 106, 023505 (2015)

[22] L. Shao and K. P. Pipe, Appl. Phys. Lett. 106, 023106 (2015).

[23] V. J. Gokhale and M. Rais-Zadeh, Sci. Rep. 4, 5617 (2014).

[24] S. A. Cummer, J. Christensen, and A. Alu, Nat. Rev. Mat. 1, 16001 (2016). 